\title{
Rescue Percutaneous Coronary Intervention for ST Elevation Myocardial Infarction After Failed Full-Dose Thrombolytic Therapy
}

National Cancer Institute

\section{Source}

National Cancer Institute. Rescue Percutaneous Coronary Intervention for ST Elevation

Myocardial Infarction After Failed Full-Dose Thrombolytic Therapy. NCI Thesaurus. Code C100008

A percutaneous coronary intervention is imperative for a myocardial infarction that presents with ST segment elevation after an unsatisfactory response to a full dose of thrombolytic therapy. (ACC) 